\title{
Delayed coupling of logistic maps
}

\author{
C. Masoller, ${ }^{1}$ Hugo L. D. de S. Cavalcante, ${ }^{2}$ and J. R. Rios Leite ${ }^{2}$ \\ ${ }^{1}$ Instituto de Física, Facultad de Ciencias, Universidad de la República, Igua 4225, Montevideo 11400, Uruguay \\ ${ }^{2}$ Departamento de Física, Universidade Federal de Pernambuco, 50670-901 Recife PE, Brazil \\ (Received 12 February 2001; revised manuscript received 19 April 2001; published 14 August 2001)
}

\begin{abstract}
We study the synchronization of logistic maps in a one-way coupling configuration. The master system is coupled to the slave system with a delay $n_{1}$, and the slave is a delayed logistic map with a delay $n_{2}$. We show that when the slave system has no delay $\left(n_{2}=0\right)$, perfectly synchronized solutions exist for strong enough coupling. In these solutions the slave variable $y$ is retarded with respect to the master variable $x$ with a retardation equal to the delay of the coupling $\left[y\left(i+n_{1}\right)=x(i)\right]$. When $n_{2} \neq 0$, a regime of generalized synchronization is observed, where $y\left(i+n_{1}\right)$ is synchronized with $x(i)$, but not completely, since the master and the slave systems obey different maps. We introduced a similarity function as an indicator of the degree of synchronization and, using a noisy master source, distinguished synchronization from noise-induced correlations.
\end{abstract}

DOI: 10.1103/PhysRevE.64.037202

PACS number(s): 05.45.Xt

Synchronization of chaotic systems has been the topic of much investigation in the last decade [1-3]. Particular attention has been paid to the inclusion of time delay, since the information exchanged among systems is delayed information [4-11]. In a pioneering work, Voss [9] recently showed that a dissipative chaotic system with a time-delayed feedback can drive a nearly identical system in such a way that the slave system anticipates the master by synchronizing with its future state. This counterintuitive behavior was shown to be robust, and a result of the interplay between delayed feedback and dissipation. Thus, it constitutes a rather universal phenomenon of nonlinear dynamics, and it has been recently found in numerical simulations of unidirectionally coupled chaotic lasers [12]. For small anticipation times, Voss has shown that anticipated synchronization also occurs in chaotic systems without a memory term in the master. Thus, this raises the question whether this behavior can be found in coupled chaotic maps with delay. In this Brief Communication we consider logistic maps coupled in a one-way delayed configuration. To the best of our knowledge, previous investigations of coupled logistic maps have considered either mutually coupled maps (see, e.g., $[13,14]$ and references therein), or a different coupling scheme [15-17].

The equations we consider are such that the master system $x$ does not have a memory term, the slave system $y$ is a delayed map, and there is also a delay in the coupling of the master to the slave map:

$$
\begin{gathered}
x(i+1)=a_{1} x(i)[1-x(i)] \\
y(i+1)=a_{2} y\left(i-n_{2}\right)\left[1-y\left(i-n_{2}\right)\right]+\eta x\left(i-n_{1}\right)[1-x(i \\
\left.\left.-n_{1}\right)\right]
\end{gathered}
$$

with $n_{1}, n_{2} \geqslant 0, i \geqslant \max \left(n_{1}, n_{2}\right)+1$, and $\eta$ is a real coefficient that controls the strength of the coupling.

If $a_{1}=a_{2}+\eta$, Eq. (2) can be rewritten as

$$
\begin{aligned}
y(i+1)= & a_{1} y\left(i-n_{2}\right)\left[1-y\left(i-n_{2}\right)\right]+\eta\left\{x\left(i-n_{1}\right)[1-x(i\right. \\
& \left.\left.\left.-n_{1}\right)\right]-y\left(i-n_{2}\right)\left[1-y\left(i-n_{2}\right)\right]\right\}
\end{aligned}
$$

From Eq. (3) it is clear that when $n_{2}=0$ synchronized solutions of the form $y\left(i-n_{2}\right)=x\left(i-n_{1}\right)$ are, in principle, possible, since in this case the equation for the slave variable $y$ becomes Eq. (1). In order to study the stability of the solution $y(i)=x\left(i-n_{1}\right)$ when $n_{2}=0$ and $a_{1}=a_{2}+\eta$ we calculate

$$
\begin{aligned}
y(i+ & 1)-x\left(i-n_{1}+1\right) \\
& =a_{2} y(i)[1-y(i)]+\left(\eta-a_{1}\right) x\left(i-n_{1}\right)\left[1-x\left(i-n_{1}\right)\right] \\
& =a_{2}\left\{y(i)[1-y(i)]-x\left(i-n_{1}\right)\left[1-x\left(i-n_{1}\right)\right]\right\} .
\end{aligned}
$$

After linearization we obtain

$$
\begin{aligned}
y(i+1)-x\left(i-n_{1}+1\right)= & a_{2}\left[1-2 x\left(i-n_{1}\right)\right][y(i) \\
& \left.-x\left(i-n_{1}\right)\right] .
\end{aligned}
$$

The synchronized solution would be stable if

$$
a_{2}\left|1-2 x\left(i-n_{1}\right)\right| \leqslant 1 \forall i \text {. }
$$

Since the master logistic map is dynamically bounded in the sense that its trajectory $x(i+1)=f(x(i))$ is constrained to the subinterval $\left[\min (x)=f(\max (x))=\left(4-a_{1}\right) a_{1}^{2} /\right.$ $\left.16, \max (x)=f(1 / 2)=a_{1} / 4\right]$, condition (6) becomes

$$
\begin{gathered}
a_{1} \leqslant 2+2 / a_{2}, \\
\left(4-a_{1}\right) a_{1}^{2} \geqslant 8-8 / a_{2} .
\end{gathered}
$$

Notice that if $a_{2} \leqslant 1$, synchronization occurs for all values of $a_{1}$ such that $0 \leqslant a_{1} \leqslant 4$. The results of the numerical simulations indicate that Eqs. (7) and (8) are sufficient conditions. Synchronization is found in a wider parameter region. Figures 1(a) and 1(b) show the plot of $y(i)$ against $x\left(i-n_{1}\right)$, for low [Fig. 1(a)] and large [Fig. 1(b)] coupling. In the first case a form of generalized synchronization is observed, since the trajectories of the two systems cover a particular region of 

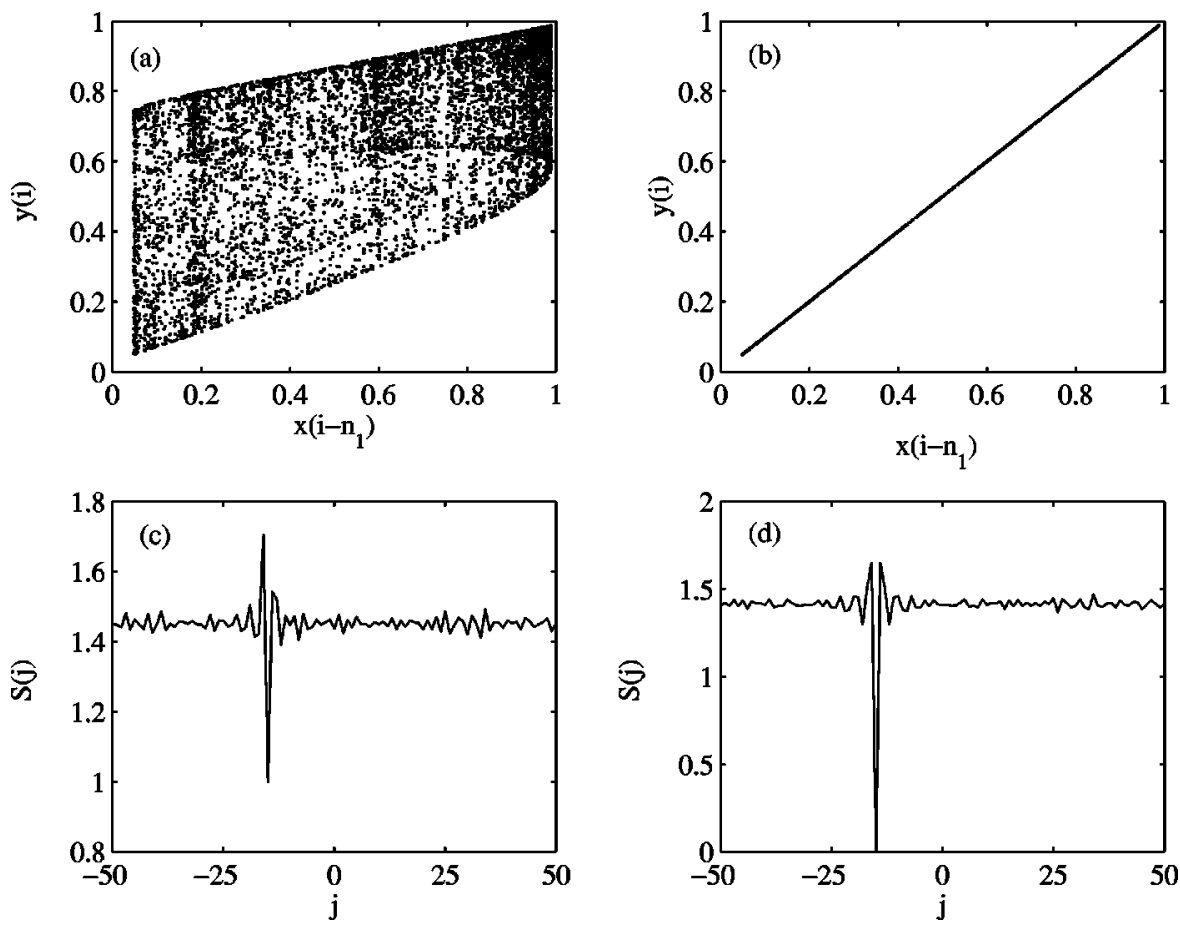

FIG. 1. (a) Phase plot of $y(i)$ vs $x\left(i-n_{1}\right)$ for $a_{1}=3.95$ when $a_{2}=3(\eta=0.95) \cdot n_{1}=15, n_{2}=0$. (b) Same as (a) but for $a_{2}=2$ ( $\eta$ $=1.95$ ). (c) and (d) Similarity function for the same parameters as (a) and (b). the phase space. In the second case perfect synchronization occurs in spite of the fact that the parameters $\left(a_{1}, a_{2}\right)$ do not verify conditions (7) and (8).

In order to quantify the degree of synchronization we calculate the similarity function [18]

$$
S^{2}(j)=\frac{\left\langle[x(i+j)-y(i)]^{2}\right\rangle}{\sqrt{\operatorname{var}[x(i)] \operatorname{var}[y(i)]}},
$$

which for perfectly synchronized solutions, $y(i)=x\left(i-n_{1}\right)$, exhibits a minimum $S=0$ for $j=-n_{1}$. Figures. 1(c) and 1(d) show the results corresponding to Figs. 1(a) and 1(b). In the case of low coupling $S(j)$ exhibits a clear minimum for $j$ $=-n_{1}$, while in the case of large coupling $S\left(-n_{1}\right)$ is zero.

The minimum of the similarity function, as a function of the parameters $\left(a_{1}, \eta / a_{1}\right)$, is shown in Fig. 2 . The parameter $a_{1}$ is varied in the region where the master system exhibits chaotic behavior, and for each value of $a_{1}, \eta$ is varied from its maximum value $\left(\eta=a_{1}\right)$ to its minimum value $(\eta=0)$. Perfect synchronization occurs in a wide range of parameter values, and it is gradually lost as the value of $\eta$ decreases. Two facts are important to notice. First, for values of $a_{1}$ such that the master system is periodic, perfect synchronization is found, even for very low (but nonzero) coupling. In the case that the slave map exhibits chaotic behavior, the coupling with the master system suppresses the chaos and with small coupling the slave synchronizes to the periodic master. In this sense, the coupling studied here can be considered a generalization of the feedback control technique employed in [19]. Second, within the region where chaos occurs for the master and the slave $\left(3.6 \leqslant a_{1}, a_{2} \leqslant 4\right)$, no synchronization was found. In this region the coupling $\eta=a_{1}-a_{2}$ must be necessarily small (at most, $|\eta|=0.4$ ), thus the degree of correlation is poor. However, the exceptions of synchronization by chaos suppression in the slave (within the periodic win- dows of the master) occur even for small coupling. In other words, the simulations show that it is easier to synchronize to a low-period periodic master than to a chaotic one. It is worth mentioning that no sensitivity to the value of $n_{1}$ was observed, indicating that synchronization is robust with respect to the initial conditions of the slave system. Synchronization occurs independently of the initial conditions of the two systems.

Perfectly synchronized solutions have only been found when $n_{2}=0$. Our numerical results show that when $n_{2} \neq 0$, for large enough coupling $y(i)$ synchronizes to $x\left(i-n_{1}\right)$ in a generalized way, since $x$ and $y$ obey different equations, and this behavior is independent of the value of $n_{2}$. As an example, Fig. 3 shows the plot of $y(i)$ against $x\left(i-n_{1}\right)$, and

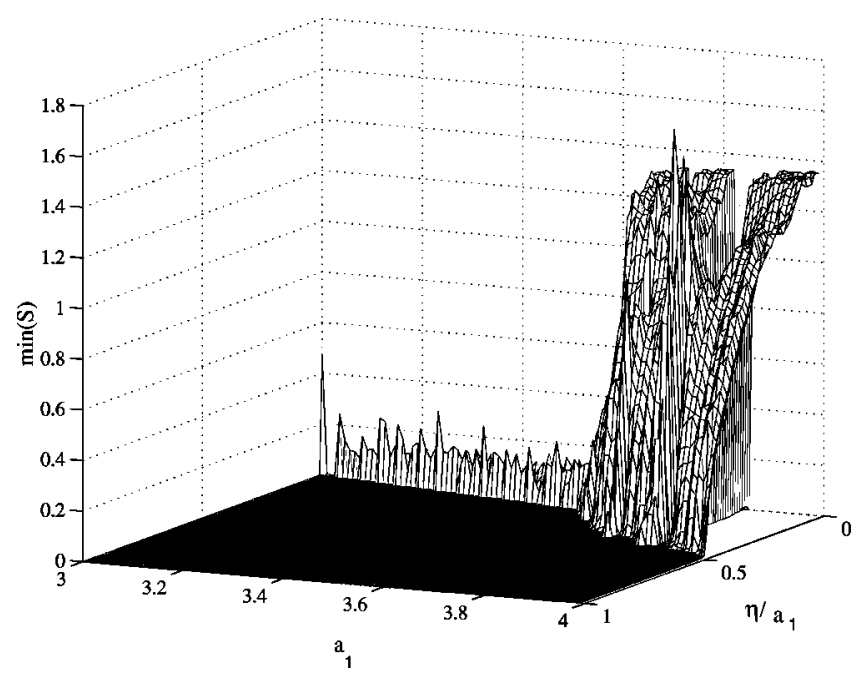

FIG. 2. Minimum of the similarity function for $n_{1}=15, n_{2}$ $=0 . a_{1}$ is varied between 3.00 and 3.99 and, for each value of $a_{1}$, $\eta / a_{1}$ is varied between 1 (maximum coupling) and 0 (no coupling). 

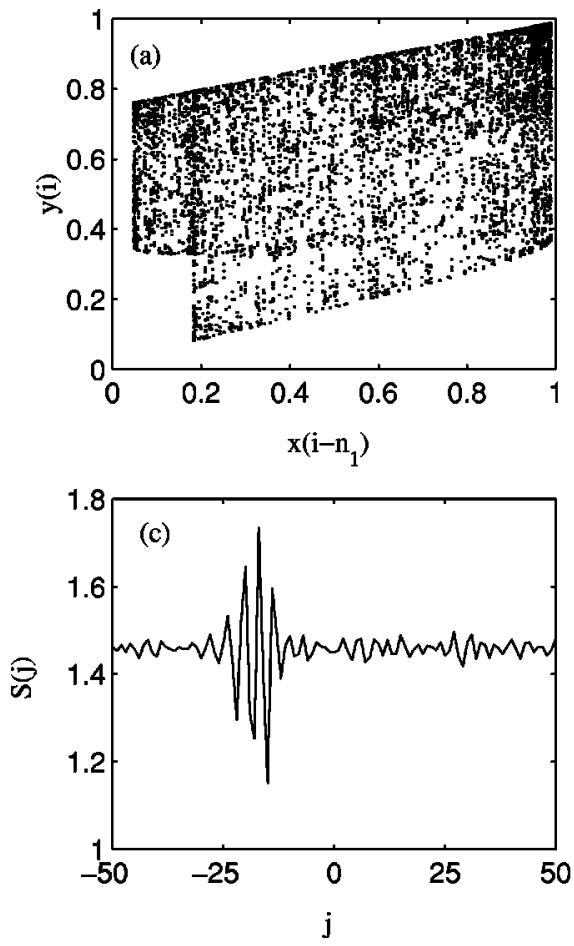
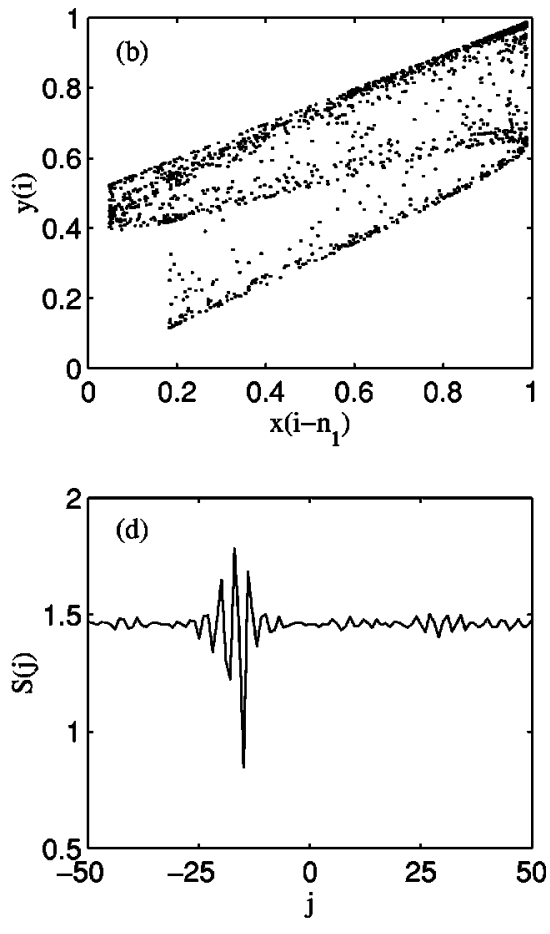

FIG. 3. (a) Phase plot for $a_{1}$ $=3.95$ when $a_{2}=3 \quad(\eta=0.95)$. $n_{1}=15, n_{2}=1$. (b) Same as (a) but for $a_{2}=2(\eta=1.95)$. (c) and (d) Similarity function for the same parameters as (a) and (b). the similarity function for the same parameters as Fig. 1, but with $n_{2}=1$. A clear minimum for $j=-n_{1}$ is observed, but synchronization is not complete. These results can be understood by noticing that Eq. (2) can be also written as

$$
y(i+1)=a_{2} y\left(i-n_{2}\right)\left[1-y\left(i-n_{2}\right)\right]+\eta x\left(i-n_{1}+1\right) / a_{1} .
$$

Therefore, in the dynamics of the slave system there is competition among the dynamics of the delayed logistic map [first term in the right-hand side of Eq. (10)], and the dynamics of the master system [second term in the right-hand side of Eq. (10)]. For large enough coupling, the dynamics of the master system dominates, and there is a strong correlation between the value $y(i+1)$ with the chaotic fluctuations of $x\left(i-n_{1}+1\right)$. This part of the correlation would survive even with a noisy master [20].

In order to confirm that the minimum of the similarity function indicates truly generalized synchronization of a deterministic origin, we have replaced $x(i)$ in Eq. (10) by a white noise, bounded by the minimum and the maximum of the master map. When the coupling is large there is a tendency of the variable $y$ to follow the random fluctuations of the noise. Figure 4 shows the results for the same parameters as Fig. 1. Notice that the minimum observed in the similarity function is much less pronounced than that in the case when the slave system is coupled the deterministic dynamics, arising from the logistic map. Similar qualitative results were obtained for noise that has the same probability density as the signal arising from the master map, showing that the noise spectrum is not important.

To summarize, we have studied the synchronization of logistic maps in a one-way coupling configuration, where the coupling has a delay $n_{1}$, and the slave system, an additional delay $n_{2}$. We have found that for strong enough coupling $y(i)$ synchronizes with $x\left(i-n_{1}\right)$ independently of the value of $n_{2}$, and synchronization is complete when $n_{2}=0$. The minimum of the similarity function as an indicator of the degree of synchronization was investigated. Using a noisy master source, a residual correlation between the master and the slave persisted. This indicates that partial, or generalized, synchronization must be carefully distinguished from noiseinduced correlation in coupled complex systems.

The authors gratefully acknowledge support from PRONEX-CNPq, Brazil, and PEDECIBA and CSIC, Uruguay.
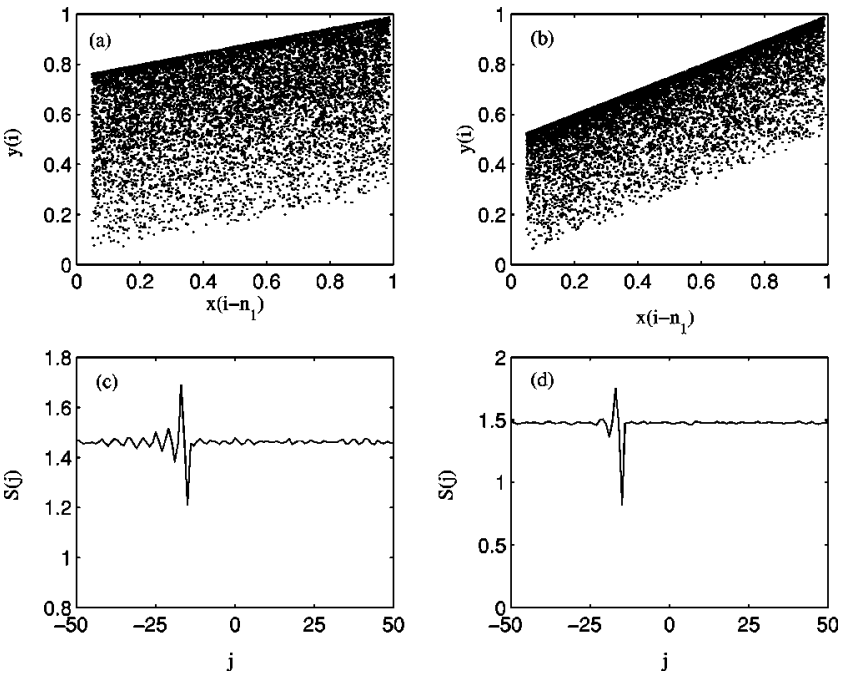

FIG. 4. (a) Phase plot with parameters as Fig. 1, but the slave system is driven by a bounded white noise as explained in the text. (b) Same as (a) but for $a_{2}=2(\eta=1.95)$. (c) and (d) Similarity function for the same parameters as (a) and (b). 
[1] H. Fujisaka and T. Yamada, Prog. Theor. Phys. 69, 32 (1983).

[2] L.M. Pecora and T.L. Carroll, Phys. Rev. Lett. 64, 821 (1990).

[3] Y. Liu, P.C. De Oliveira, M.B. Danailov, and J.R. Rios Leite, Phys. Rev. A 50, 3464 (1994).

[4] M.J. Bunner and W. Just, Phys. Rev. E 58, R4072 (1998).

[5] K. Pyragas, Phys. Rev. E 58, 3067 (1998); Int. J. Bifurcation Chaos Appl. Sci. Eng. 8, 1839 (1998).

[6] S. Kim, S. Hee Park, and H.-B. Pyo, Phys. Rev. Lett. 82, 1620 (1999).

[7] R. He and P.G. Vaidya, Phys. Rev. E 59, 4048 (1999).

[8] Y. Hayakawa and Y. Sawada, Phys. Rev. E 61, 5091 (2000).

[9] H.U. Voss, Phys. Rev. E 61, 5115 (2000); Phys. Lett. A 279, 207 (2001).

[10] D.H. Zanette, Phys. Rev. E 62, 3167 (2000).

[11] C. Masoller, Phys. Rev. Lett. 86, 2782 (2001).
[12] D.V. Ramana Reddy, A. Sen, and G.L. Johnston, Phys. Rev. Lett. 85, 3381 (2000).

[13] V. Astakhov, A. Shabunin, T. Kapitaniak, and V. Anishchenko, Phys. Rev. Lett. 79, 1014 (1997).

[14] Yu.L. Maistrenko, A. Popovich, and E. Mosetilde, Phys. Rev. E 57, 2713 (1998).

[15] O. Tai, C. Skorupka, and L.M. Pecora, Phys. Lett. A 187, 175 (1994).

[16] M.K. Ali, Phys. Rev. E 55, 4804 (1997).

[17] O. Morgul, Phys. Lett. A 247, 391 (1998).

[18] M.G. Rosenblum, A.S. Pikovsky, and J. Kurths, Phys. Rev. Lett. 78, 4193 (1997).

[19] J. Escalona and P. Parmananda, Phys. Rev. E 61, 5987 (2000).

[20] S.Y. Choi and E.K. Lee, Phys. Lett. A 205, 173 (1995). 